\title{
Fatores prognósticos e evolução da função ventricular em 5 anos de seguimento da ventriculectomia parcial esquerda no tratamento da cardiomiopatia dilatada
}

\author{
Luiz Felipe P. MOREIRA*, Fernando BACAL*, Anderson BENÍCIO*, Edimar A. BOCCHI*, \\ Maria L. HIGUCHI*, Noedir A. G. STOLF*, Sérgio Almeida de OLIVEIRA*
}

RBCCV 44205-556

\begin{abstract}
Moreira L F P, Bacal F, Benício A, Bocchi E A, Higuchi M L, Stolf N A G, Oliveira S A - Fatores prognósticos e evolução da função ventricular em 5 anos de seguimento da ventriculectomia parcial esquerda no tratamento da cardiomiopatia dilatada. Rev Bras Cir Cardiovasc 2001; 16(4): 275-88.
\end{abstract}

RESUMO: Objetivo: Nesta investigação, os resultados tardios da ventriculectomia parcial esquerda, associada à correção da insuficiência das valvas atrioventriculares, foram estudados em 43 pacientes portadores de cardiomiopatia dilatada.

Casuística e Métodos: Os pacientes estavam em classe funcional III (18) ou IV (25) no pré-operatório, sendo que 7 pacientes foram operados na vigência de choque cardiogênico. A redução cirúrgica do volume do ventrículo esquerdo (VE) foi associada à anuloplastia mitral em 32 pacientes e à substituição daquela valva em 3. Em 10 pacientes, também foi realizada plastia de valva tricúspide. Doze pacientes foram submetidos ao implante de desfibriladores automáticos.

Resultados: Ocorreram $9(20,9 \%)$ óbitos hospitalares. O tempo de seguimento pós-operatório variou entre dois e 68 meses, com média de 34,2 meses. Aos seis meses de seguimento, 8 pacientes estavam em classe funcional I, $13 \mathrm{em}$ classe II, $3 \mathrm{em}$ classe III e $1 \mathrm{em}$ classe IV $(p<0,001)$. Por outro lado, outros 19 pacientes faleceram no seguimento tardio, sendo observados índices de sobrevida de 58 (?7\% em um ano, de 48 (?7\% aos dois anos e de 32?(?8\% aos cinco anos. A regressão logística mostrou que a sobrevida nos primeiros seis meses foi influenciada pelo diâmetro das fibras miocárdicas. A análise da sobrevida tardia através do modelo proporcional de Cox mostrou que a classe funcional IV e o nível elevado de nor-adrenalina sérica no pré-operatório estavam relacionados a maior mortalidade. Quando a análise também incluiu variáveis anatomopatológicas, a existência de apoptose das células miocárdicas e o maior diâmetro das fibras miocárdicas no pré-operatório foram identificados como únicos fatores independentes de pior prognóstico tardio. Pacientes operados em classe funcional III ou IV apresentaram índices de sobrevida de 60,6?(?11,6\% e de 14,4?(?8,2\% em cinco anos, respectivamente. Na presença de apoptose das células miocárdicas, a sobrevida dos pacientes foi de 8,3?(?7,9\% no mesmo período, contra $63,1 ?(? 11 \%$ na ausência dessa alteração. Em relação à função ventricular, os benefícios observados após a ventriculectomia parcial não se mantiveram tardiamente. Houve redilatação progressiva do ventrículo esquerdo, associada à queda concomitante dos valores de sua fração de ejeção.

Conclusão: A ventriculectomia parcial esquerda, associada, quando necessário, à correção da insuficiência das valvas atrioventriculares, melhora a condição clínica e a função ventricular de pacientes com cardiomiopatia dilatada idiopática. Por outro lado, os resultados tardios deste procedimento são limitados pelo grau de comprometimento das fibras miocárdicas e pela severidade do comprometimento funcional dos pacientes no pré-operatório. Além disso, os seus benefícios a função ventricular são comprometidos pela possibilidade de redilatação do ventrículo esquerdo.

DESCRITORES: Miocardiopatia congestiva, cirurgia. Procedimentos cirúrgicos cardíacos, métodos. Ventrículo cardíaco, cirurgia.

Trabalho realizado no Instituto do Coração do Hospital das Clínicas da Faculdade de Medicina da Universidade de São Paulo. São Paulo, SP, Brasil. Apresentado ao 28을 Congresso Nacional de Cirurgia Cardíaca. Ouro Preto, MG, 5 a 7 de abril de 2001.

* Do Instituto do Coração do Hospital das Clínicas da Faculdade de Medicina da Universidade de São Paulo.

Endereço para correspondência: Luiz Felipe P. Moreira. Av. Dr. Enéas de Carvalho Aguiar, 44 - 2ªndar. São Paulo, SP, Brasil. CEP: $05403-000$

Tel.: (11) 3069-5075 / Fax:(11) 282-2354. e-mail: dcimoreira@incor.usp.br 
Moreira L F P, Bacal F, Benício A, Bocchi E A, Higuchi M L, Stolf N A G, Oliveira S A - Fatores prognósticos e evolução da função ventricular em 5 anos de seguimento da ventriculectomia parcial esquerda no tratamento da cardiomiopatia dilatada. Rev Bras Cir Cardiovasc 2001; 16(4): 275-88.

\section{INTRODUÇÃO}

A redução cirúrgica do volume do ventrículo esquerdo (VE) foi proposta por BATISTA et al. (1), em 1996, sendo atualmente avaliada em diversos centros de cirurgia cardíaca em todo o mundo. Este procedimento, conhecido com o nome de ventriculectomia parcial esquerda, tem sido geralmente associado à plastia ou à substituição das valvas atrioventriculares, no tratamento de pacientes portadores de cardiomiopatia dilatada.

Os resultados do emprego clínico da ventriculectomia parcial têm sido, no entanto, controversos. Vários estudos demonstram a melhora da função ventricular esquerda e dos sintomas de insuficiência cardíaca após a realização deste procedimento (2-15). Por outro lado, incidências elevadas de óbitos relacionados à progressão da insuficiência cardíaca ou a arritmias têm sido relatadas nos primeiros meses de seguimento pós-operatório, na maioria dos estudos clínicos $(2-5,7,12,14)$, representando grande limitação para o emprego rotineiro desta técnica. Paralelamente, faltam informações sobre os fatores relacionados com o prognóstico imediato e tardio após a realização do procedimento.

O presente estudo foi desenvolvido com a finalidade de analisar a influência de parâmetros pré, intra e pós-operatórios imediatos sobre os resultados imediatos e tardios da ventriculectomia parcial esquerda em pacientes com cardiomiopatia dilatada idiopática. Nessa população, este procedimento foi associado, quando necessário, à correção da insuficiência das valvas atrioventriculares.

\section{CASUÍSTICA E MÉTODOS}

\section{População Estudada}

Nesta investigação foram estudados, prospectivamente, 43 pacientes portadores de cardiomiopatia dilatada idiopática, que foram submetidos à ventriculectomia parcial esquerda, associada, quando necessário, à correção da insuficiência das valvas atrioventriculares. Os procedimentos foram realizados no Instituto do Coração do Hospital das Clínicas da Faculdade de Medicina da Universidade de São Paulo, no período de abril de 1995 a março de 1999.

A indicação da ventriculectomia parcial esquerda foi baseada na existência de limitação funcional importante, apesar da otimização da terapêutica medicamentosa com doses máximas de diuréticos, drogas inibidoras da enzima de conversão ou vasodilatadores e, eventualmente, de betabloqueadores. Os pacientes também apresentavam comprometimento importante da função ventricular esquerda, caracterizado pela fração de ejeção do $\mathrm{VE} \leq 25 \%$, quando calculada pela angiografia com radioisótopos, e pela existência de hipertensão em território pulmonar.

Os pacientes apresentavam contra-indicações médicas ou psicossociais ao transplante cardíaco ou haviam recusado a realização daquele procedimento. Todos assinaram o formulário de consentimento, redigido de acordo com as normas da Comissão Científica e de Ética do Instituto do Coração.

Trinta e seis pacientes eram do sexo masculino e 7 pacientes do sexo feminino. A idade dos pacientes variou entre 28 e 72 anos, com média de 45,5 anos. Todos os pacientes referiam sintomas de insuficiência cardíaca por um período superior a um ano antes da indicação do tratamento cirúrgico, tendo sido hospitalizados no mínimo uma vez nos últimos seis meses por descompensação do quadro clínico. Dezoito pacientes estavam em classe funcional III e 25 pacientes em classe funcional IV, segundo os critérios da NYHA. Quatorze pacientes haviam utilizado drogas inotrópicas endovenosas durante a internação na qual foi realizada a operação, sendo que 7 pacientes foram operados na vigência do suporte farmacológico para tratamento do choque cardiogênico. A dosagem plasmática dos níveis de nor-epinefrina mostrou valores que variaram entre 288 e $1182 \mathrm{pg} \cdot \mathrm{ml}^{-1}$, com média de $611 ?(? 241$ pg.ml-1).

O eletrocardiograma revelou que 39 pacientes estavam em ritmo sinusal antes da ventriculectomia parcial e que apenas 4 pacientes estavam em fibrilação atrial. Esse exame mostrou ainda que 29 pacientes apresentavam bloqueio completo de ramo esquerdo. A existência de potencial tardio significativo foi evidenciada em 12 dos 32 pacientes submetidos a eletrocardiografia de alta resolução. Episódios de taquicardia ventricular não sustentada foram documentados pela eletrocardiografia de 24 horas em 22 dos 33 pacientes estudados por esse método.

Os valores das variáveis pré-operatórias obtidas através da avaliação da função ventricular encontram-se na Tabela 1. Segundo a ecocardiografia com Doppler, 38 pacientes apresentavam insuficiência da valva atrioventricular esquerda, a qual foi quantificada como leve em 18 pacientes, como moderada em 15 pacientes e como importante em 5 pacientes. Onze pacientes também apre- 
Moreira L F P, Bacal F, Benício A, Bocchi E A, Higuchi M L, Stolf N A G, Oliveira S A - Fatores prognósticos e evolução da função ventricular em 5 anos de seguimento da ventriculectomia parcial esquerda no tratamento da cardiomiopatia dilatada. Rev Bras Cir Cardiovasc 2001; 16(4): 275-88.

\section{TABELA 1}

PARÂMETROS DA FUNÇÃO VENTRICULAR NO PRÉ-OPERATÓRIO

\section{Estudo Ecocardiográfico}

Diâmetro diastólico do VE $(\mathrm{mm})$

$83,2 \pm 8,7$

Encurtamento segmentar do VE (\%)

$11,8 \pm 2,6$

\section{Angiografia com Radioisótopos}

Volume diastólico do VE (ml)

$552 \pm 182$

Fração de ejeção do VE (\%)

Fração de ejeção do VD (\%)

$16,6 \pm 4,7$

$21,1 \pm 6,3$

\section{Cateterismo Cardíaco Direito}

Pressão média de átrio direito *

Pressão média de artéria pulmonar*

$9,4 \pm 4,9$

Pressão média de capilar pulmonar*

$35,5 \pm, 91$

Pressão arterial média *

$24,1 \pm 6$

Índice cardíaco (I. $\left.\mathrm{min}^{-1} \mathrm{~m}^{-2}\right)$

Índice sistólico $\left(\mathrm{ml}^{\mathrm{b}} \mathrm{bat}^{-1} \cdot \mathrm{m}^{-2}\right)$

$80,5 \pm 11,1$

$2,12 \pm 0,46$

Índice de trabalho sistólico do VE **

$23,2 \pm 5,9$

Resistência vascular pulmonar ***

$18,1 \pm 7,1$

$241 \pm 132$

$\mathrm{VE}=$ Ventrículo esquerdo; $\mathrm{VD}=$ Ventrículo direito; ${ }^{*}=(\mathrm{mmHg})$; ${ }^{* *}=\left(\mathrm{g} \cdot \mathrm{m} \cdot \mathrm{m}^{-2}\right) ;{ }^{* *}=$ dyn.s.cm ${ }^{-5}$.

sentavam insuficiência significativa da valva atrioventricular direita, sendo quantificada como moderada em 7 pacientes e como importante em 4 . O cateterismo cardíaco revelou ainda que nenhum dos pacientes apresentava obstruções significativas (? 70\%) nas artérias coronárias.

\section{Procedimento Cirúrgico}

A ventriculectomia parcial esquerda foi realizada como procedimento isolado ou associado a plastia ou substituição das valvas atrioventriculares. Os primeiros 35 pacientes foram operados em normotermia e com o coração batendo. Nos últimos 8 casos, os procedimentos foram realizados sob hipotermia moderada a 27??C e parada anóxica induzida pela infusão de solução cardioplégica sangüínea, resfriada a 4 ??C.

A ressecção parcial do VE foi realizada de acordo com a técnica descrita anteriormente $(1,16)$. A valva mitral foi abordada através do átrio esquerdo e o anel mitral foi reduzido pela plicatura do folheto posterior com suturas ancoradas em tira de pericárdio bovino. Quando os músculos papilares interferiam na obtenção de uma ressecção efetiva do miocárdio ventricular, esses músculos foram ressecados e a valva mitral foi substituída por bioprótese de pericárdio bovino. Os pacientes com graus moderados ou importantes de insuficiência da valva tricúspide foram submetidos a plástica do tipo De Vega.
Amiodarona foi empregada rotineiramente nas primeiras duas semanas de pós-operatório, sendo mantida posteriormente em pacientes que apresentaram episódios de taquicardia ventricular sustentada ou com o objetivo de diminuir a freqüência cardíaca em pacientes com fibrilação atrial. Desfibriladores automáticos foram implantados rotineiramente nos pacientes que apresentaram episódios de taquicardia ventricular sustentada ou de fibrilação ventricular no período pós-operatório imediato.

\section{Avaliação dos Parâmetros Relacionados com a Sobrevida}

Com o objetivo de avaliar a influência do grau de comprometimento dos pacientes no pré-operatório sobre a sobrevida tardia após a ventriculectomia parcial esquerda, foram analisados parâmetros clínicos, eletrocardiográficos, ecocardiográficos, radioisotópicos e hemodinâmicos. O diâmetro das fibras miocárdicas e a presença de apoptose de miócitos cardíacos, obtidos através do estudo anatomopatológico realizado nos fragmentos miocárdicos ressecados no procedimento cirúrgico (16), também foram considerados como parâmetros pré-operatórios. A influência dos aspectos técnicos da ventriculectomia parcial esquerda sobre a sobrevida no pós-operatório também foi estudada.

\section{Análise Estatística}

Os índices de mortalidade e de eventos ocorridos durante o seguimento pós-operatório da ventriculectomia parcial esquerda foram determinados pelo método de Kaplan-Meier. As curvas obtidas foram analisadas através de regressão não linear.

A associação de variáveis pré, intra e pósoperatórias com a sobrevida ou eventos mórbidos foi avaliada através da análise isolada das variáveis, utilizando-se o teste $\mathbf{t}$ de Student para amostras não pareadas na análise das variáveis quantitativas e o teste exato de Fisher para as variáveis qualitativas. Variáveis com valor de $\mathbf{p}$ menor do que 0,10 através da análise isolada foram submetidas à análise multivariada por regressão logística em degraus e à análise de risco proporcional de Cox. As curvas de sobrevida e de eventos obtidas a partir das variáveis consideradas fatores independentes de prognóstico foram comparadas através do teste de log-rank.

Todos os cálculos foram realizados empregando-se o programa de análise estatística por computador SPSS for Windows 9.0. Foram considerados significantes os valores com probabilidade inferior a 0,05 . 
Moreira L F P, Bacal F, Benício A, Bocchi E A, Higuchi M L, Stolf N A G, Oliveira S A - Fatores prognósticos e evolução da função ventricular em 5 anos de seguimento da ventriculectomia parcial esquerda no tratamento da cardiomiopatia dilatada. Rev Bras Cir Cardiovasc 2001; 16(4): 275-88.

\section{RESULTADOS}

\section{Dados Operatórios}

Oito pacientes foram submetidos apenas à ventriculectomia parcial esquerda, enquanto que este procedimento foi associado a anuloplastia mitral em 32 pacientes e a substituição daquela valva em 3 pacientes. Em 10 dos pacientes que foram submetidos a correção da valvopatia mitral, também foi realizada plastia de valva tricúspide. A extensão longitudinal do fragmento ressecado do miocárdio ventricular foi de $10,9 \pm ? 1,7 \mathrm{~cm}$ e a largura da ressecção realizada de $5,1 ?(? ? 0,9 \mathrm{~cm}$. A análise morfométrica do miocárdio ventricular mostrou um diâmetro médio das fibras miocárdicas 22,3?(?2,9 microns. Já a pesquisa de apoptose em células miocárdicas mostrou a presença dessa alteração em 16 dos 31 pacientes pesquisados.

O ecocardiograma transesofágico foi realizado após o término da circulação extracorpórea e mostrou ausência de insuficiência mitral residual em 31 pacientes e grau leve de regurgitação em 12. Todos os pacientes receberam medicações inotrópicas positivas e vasodilatadores ao final do procedimento cirúrgico, sendo necessária a implantação do balão intra-aórtico em 17 (39,5\%) doentes.

\section{Evolução no Pós-Operatório Imediato}

O suporte inotrópico endovenoso foi mantido geralmente por uma semana após a operação e o balão intra-aórtico foi normalmente retirado no segundo ou terceiro dia de pós-operatório. Dois pacientes apresentaram disfunção ventricular refratária ao tratamento farmacológico e ao uso do balão intra-aórtico, sendo realizada a inserção de bomba centrífuga ou de dispositivo pneumático de assistência ventricular para o VE, dois dias após a ventriculectomia parcial. Outro paciente apresentou deiscência da anuloplastia mitral e foi reoperado para substituição da valva mitral no quinto dia de pós-operatório. Episódios de taquicardia ventricular sustentada ocorreram em 9 $(20,9 \%)$ pacientes nas primeiras quatro semanas de seguimento.

Nove $(20,9 \%)$ pacientes faleceram durante 0 período hospitalar. As causas de óbito foram: choque cardiogênico em 4 pacientes; falência biventricular no paciente mantido em assistência ventricular esquerda com bomba centrífuga; falência de múltiplos órgãos no paciente submetido ao implante de dispositivo pulsátil de assistência ventricular esquerda; septicemia e choque cardiogênico no paciente reoperado para correção da deiscência da anuloplastia mitral; taquicardia ventricular incessante em 1 paciente; e sangramento associado a coagulação intravascular disseminada em outro paciente.

\section{Seguimento Pós-Operatório Tardio}

Nenhum dos pacientes foi perdido para seguimento durante o período desta investigação. O tempo de acompanhamento pós-operatório variou entre dois e 68 meses, com média de $34,2 \pm$ ?24,8 meses, perfazendo um total de 97,3 pacientes-ano.

Aos seis meses de pós-operatório, 8 pacientes estavam em classe funcional I, 13 pacientes em classe funcional II, 3 pacientes em classe funcional III e 1 paciente em classe funcional IV $(p<0,001$ em relação ao pré-operatório). No entanto, 10 pacientes foram re-hospitalizados durante o primeiro ano de seguimento, devido a progressão de insuficiência cardíaca e outros 3 devido a episódios de taquicardia ventricular sustentada. $O$ transplante cardíaco foi indicado em 5 desses pacientes, sendo realizado em caráter de urgência em 1 deles, sete meses após a ventriculectomia parcial.

Após o primeiro ano de seguimento, 9 pacientes necessitaram re-hospitalizações por descompensação da insuficiência cardíaca, em uma incidência de 0,2 internações por paciente-ano. Três pacientes portadores de desfibriladores automáticos implantáveis apresentaram episódios de taquicardia ventricular sustentada, que foram revertidos através de desfibrilação elétrica aos 30,33 e 40 meses de seguimento, respectivamente.

Nove pacientes faleceram durante os primeiros seis meses de seguimento e outros dez óbitos ocorreram após o primeiro ano de pós-operatório. A causa dos óbitos que ocorreram nos primeiros seis meses estava relacionada à progressão de insuficiência cardíaca em 5 pacientes. Quatro pacientes faleceram subitamente ou em decorrência de arritmias ventriculares sustentadas. Os óbitos observados após um ano de seguimento ocorreram por progressão de insuficiência cardíaca em 6 pacientes e 4 doentes faleceram subitamente após aquele período. Seis dos pacientes que faleceram por arritmia estavam em uso de amiodarona e nenhum deles era portador de desfibrilador automático implantável.

A curva de sobrevida, calculada pelo método de Kaplan-Meier (Gráfico 1), mostra índices de sobrevida de 58,1 (? 7,5\% no primeiro ano após a ventriculectomia parcial esquerda, de 48,4 (?7,6\% em dois anos, de 45,9 (?7,6\% em três anos, de 40,8 (?7,6\% em quatro anos e de 34,1 (? 7,7\% aos cinco anos de seguimento. A primeira fase da curva se refere aos seis primeiros meses de pós-operatório e foi a fase de maior mortalidade. No período subseqüente, o risco aproximado de mortalidade foi de 12,2 eventos por 100 paciente-ano. 
Moreira L F P, Bacal F, Benício A, Bocchi E A, Higuchi M L, Stolf N A G, Oliveira S A - Fatores prognósticos e evolução da função ventricular em 5 anos de seguimento da ventriculectomia parcial esquerda no tratamento da cardiomiopatia dilatada. Rev Bras Cir Cardiovasc 2001; 16(4): 275-88.

GRÁFICO 1

CURVA DE SOBREVIDA (KAPLAN-MEIER) APÓS A VENTRICULECTOMIA PARCIAL ESQUERDA. OS NÚMEROS ENTRE PARÊNTESES INDICAM OS PACIENTES EM RISCO EM CADA PERÍODO ANALISADO. AS PROBABILIDADES SÃO APRESENTADAS COM O LIMITE DE CONFIANÇA DE 70\%

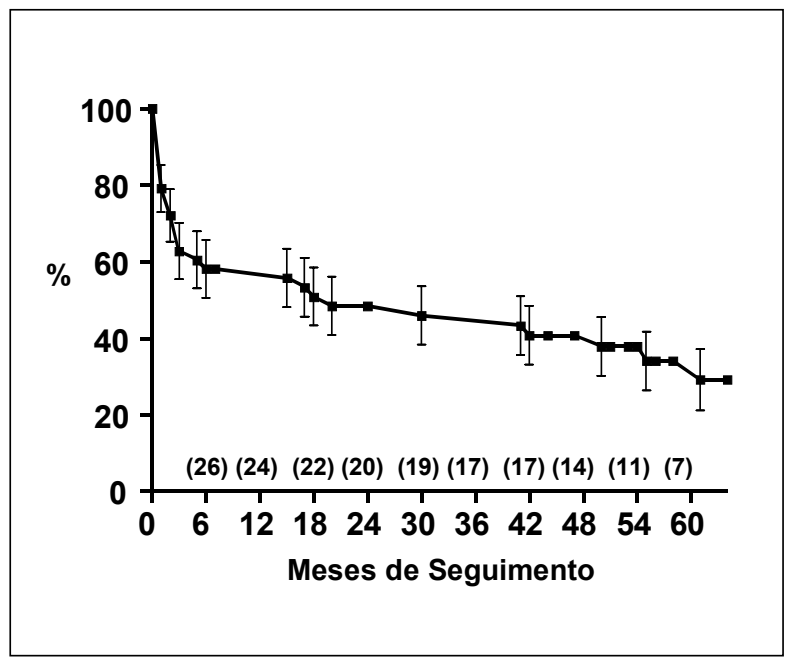

GRÁFICO 2

CURVAS DE INCIDÊNCIA DE ÓBITOS POR PROGRESSÃO DE INSUFICIÊNCIA CARDÍACA CONGESTIVA (ICC) E POR ARRITMIAS OU MORTE SÚBITA (MS) NO SEGUIMENTO PÓS-OPERATÓRIO DA VENTRICULECTOMIA PARCIAL ESQUERDA

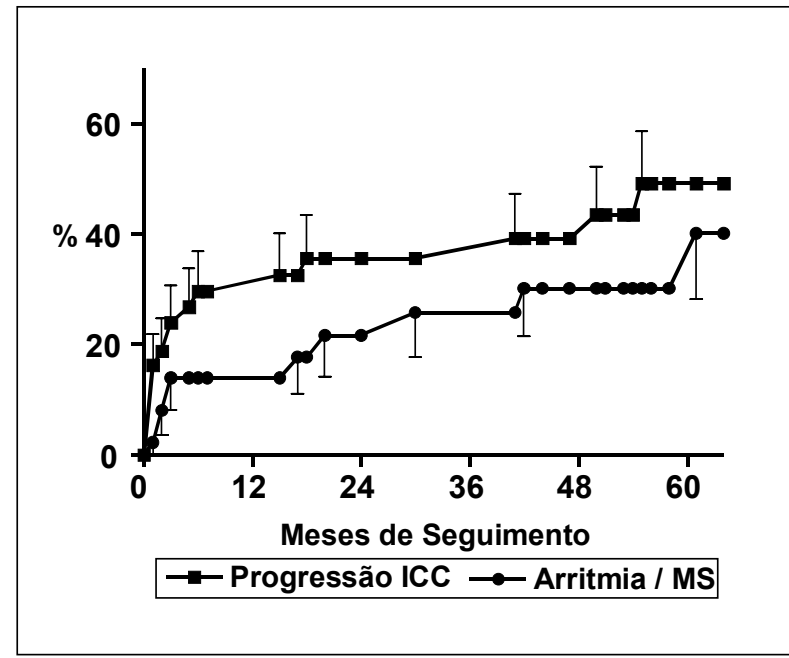

TABELA 2

ANÁLISE ISOLADA DOS PARÂMETROS PRÉ-OPERATÓRIOS RELACIONADOS COM A SOBREVIDA EM SEIS MESES DE SEGUIMENTO APÓS A VENTRICULECTOMIA PARCIAL ESQUERDA

\begin{tabular}{lccc}
\hline & SOBREVIVENTES & ÓBITOS & p \\
\hline Extensão longitudinal da ressecção miocárdica $(\mathrm{cm})$ & $10,3 \pm 1,2$ & $11,6 \pm 1,9$ & $\mathrm{p}=0,015$ \\
Diâmetro das fibras miocárdicas (microns) & $21,5 \pm 2,7$ & $23,4 \pm 2,8$ & $\mathrm{p}=0,041$ \\
Nível plasmático de nor-epinefrina (pg. $\mathrm{ml}^{-1}$ ) & $549 \pm 201$ & $696 \pm 268$ & $\mathrm{p}=0,046$ \\
Fração de ejeção de ventrículo esquerdo $(\%)$ & $17,7 \pm 4,9$ & $15,1 \pm 4,2$ & $\mathrm{p}=0,081$ \\
Diâmetro diastólico de ventrículo esquerdo $(\mathrm{mm})$ & $81,2 \pm 6,9$ & $85,9 \pm 10,4$ & $\mathrm{p}=0,086$ \\
\hline
\end{tabular}

Valores apresentados em média \pm desvio padrão.

À semelhança da curva de sobrevida, o Gráfico 2 mostra que tanto os óbitos relacionados a progressão da insuficiência cardíaca, como aqueles que ocorreram subitamente ou por arritmias ventriculares apresentaram incidências elevadas nos primeiros meses de seguimento. Após o sexto mês de pósoperatório, a incidência aproximada de óbitos por insuficiência cardíaca foi de 7,3 eventos por 100 paciente-ano, enquanto que os óbitos associados a alterações do ritmo cardíaco apresentaram incidência de 4,9 eventos por 100 paciente-ano.

\section{Fatores Relacionados com a Sobrevida}

A análise isolada das variáveis pré-operatórias estudadas identificou a existência de relação significativa entre os seguintes parâmetros e a sobrevida nos primeiros seis meses de seguimento: diâmetro das fibras miocárdicas, nível plasmático de norepinefrina, fração de ejeção do ventrículo esquerdo (VE) e diâmetro diastólico do VE (Tabela 2). $\mathrm{Na}$ mesma tabela observamos que, em relação aos parâmetros estudados no intra e no pós-operatório imediato, foi identificada relação significativa das variáveis extensão longitudinal da ressecção miocárdica, variação porcentual do diâmetro diastólico do VE e variação porcentual da fração de ejeção do VE com a sobrevida no mesmo período.

Quando os parâmetros pré-operatórios foram submetidos a análise multivariada por regressão logística em degraus, apenas o grau de hipertrofia das células miocárdicas foi identificado como fator independente de prognóstico, apresentando um nível de significância de 0,042 . O risco de mortalidade no período analisado se eleva na presença de valores progressivamente maiores do diâmetro das fibras miocárdicas, em uma 
Moreira L F P, Bacal F, Benício A, Bocchi E A, Higuchi M L, Stolf N A G, Oliveira S A - Fatores prognósticos e evolução da função ventricular em 5 anos de seguimento da ventriculectomia parcial esquerda no tratamento da cardiomiopatia dilatada. Rev Bras Cir Cardiovasc 2001; 16(4): 275-88.

TABELA 3

\begin{tabular}{lccc}
\hline \multicolumn{4}{c}{ ANÁLISE ISOLADA DOS PARÂMETROS PRÉ-OPERATÓRIOS RELACIONADOS COM } \\
A SOBREVIDA NO PÓS-OPERATÓRIO TARDIO \\
\hline & SOBREVIVENTES & ÓBITOS & p \\
\hline Apoptose de células miocárdicas (n/campo) & $0,41 \pm 0,9$ & $7,2 \pm 9,3$ & $\mathrm{p}=0,005$ \\
Diâmetro das fibras miocárdicas (microns) & $20,9 \pm 2,3$ & $23,1 \pm 2,9$ & $\mathrm{p}=0,024$ \\
Classe funcional & III (10) IV (5) & III (8) IV (20) & $\mathrm{p}=0,024$ \\
Pressão média de capilar pulmonar (mmHg) & $21,1 \pm 6,3$ & $25,4 \pm 5,6$ & $\mathrm{p}=0,026$ \\
Classe funcional & III (9) IV (6) & III (9) IV (19) & $\mathrm{p}=0,057$ \\
Encurtamento segmentar do ventrículo esquerdo (\%) & $12,8 \pm 2,3$ & $11,3 \pm 2,6$ & $\mathrm{p}=0,059$ \\
Nível plasmático de nor-epinefrina $\left(\mathrm{pg} \cdot \mathrm{ml}^{-1}\right.$ ) & $528 \pm 184$ & $658 \pm 259$ & $\mathrm{p}=0,095$ \\
Índice de trabalho sistólico do ventrículo esquerdo $\left(^{*}\right)$ & $20,9 \pm 8,9$ & $17,1 \pm 5,5$ & $\mathrm{p}=0,097$ \\
\hline
\end{tabular}

Valores apresentados em média \pm desvio padrão; $\left({ }^{*}\right)=\mathrm{g} \cdot \mathrm{m} \cdot \mathrm{m}^{-2}$.

razão de probabilidade de $1,3 \pm$ ? 0,2 vezes por aumento unitário desse parâmetro. A inclusão dos parâmetros intra e pós-operatórios imediatos na análise não identificou nenhum outro fator independente de prognóstico, embora o nível de significância para a variação porcentual da fração de ejeção do VE e para a extensão longitudinal da ressecção miocárdica fossem de 0,051 e de 0,059, respectivamente.

A Tabela 3 mostra os resultados do estudo univariado dos parâmetros pré-operatórios associados com a sobrevida após a ventriculectomia parcial, quando os dados do seguimento tardio são incluídos na análise. Por outro lado, nenhum fator intra ou pósoperatório imediato foi relacionado com a sobrevida global pela análise isolada das variáveis.

Através da análise de risco proporcional de Cox, a classe funcional e o nível plasmático de norepinefrina foram identificados como fatores independentes de prognóstico no pós-operatório tardio da ventriculectomia parcial, com níveis de significância de 0,0218 e 0,0397, respectivamente. Esse fato ocorreu quando foram analisados apenas os parâmetros com valores completos. Quando o modelo de risco também incluiu as variáveis anatomopatológicas, cujos valores completos foram obtidos em 31 pacientes, a existência de apoptose de miócitos cardíacos e o diâmetro das fibras miocárdicas passaram a ser os únicos fatores independentes de prognóstico. Essas variáveis apresentaram níveis de significância de 0,0066 e 0,0146, respectivamente.

As curvas de sobrevida obtidas a partir dos quatro fatores de prognóstico identificados pelos modelos de risco proporcional de Cox são apresentadas nas Gráficos 3, 4, 5 e 6 . Essas curvas mostram o impacto significativo desses fatores sobre a sobrevida no pósoperatório tardio da ventriculectomia parcial.

\section{Análise da Função Ventricular}

Os dados obtidos pela angiografia com radioisótopos estão na Tabela 4. A fração de ejeção do VE se elevou logo após a ventriculectomia parcial de 16,8 (??4,7\% para 23,9 $(? 8,2 \% \quad(p<0,01)$ os seus valores tenderam a manter até quatro anos de seguimento em torno de $21 \%$. Por outro lado, a diminuição significativa do volume diastólico do VE, documentada logo após o procedimento, se manteve apenas durante 0 primeiro ano de pósoperatório. Depois daquele período, este parâmetro tendeu a se elevar e retornou aos valores préoperatórios a partir dos três anos de seguimento.

A avaliação hemodinâmica mostrou que elevações significativas do índice sistólico, do índice cardíaco e da pressão arterial sistêmica ocorreram, associadas à queda significativa das pressões em território pulmonar, logo após a ventriculectomia parcial esquerda (Tabela 5). No seguimento tardio, apesar da manutenção das alterações positivas do índice cardíaco, do índice sistólico e da pressão arterial sistêmica, as pressões pulmonares tenderam a se elevar progressivamente.

\section{COMENTÁRIOS}

O emprego da ventriculectomia parcial esquerda, como uma alternativa ao transplante cardíaco, no tratamento de pacientes portadores de cardiomiopatia dilatada tem sido investigado com grande interesse em centros nacionais e internacionais, cuja experiência inclui, atualmente, mais de mil casos operados em todo o mundo $(2,4,6-11,13,15)$. A interpretação dos resultados obtidos em vários estudos, no entanto, é limitada pela existência de 
Moreira L F P, Bacal F, Benício A, Bocchi E A, Higuchi M L, Stolf N A G, Oliveira S A - Fatores prognósticos e evolução da função ventricular em 5 anos de seguimento da ventriculectomia parcial esquerda no tratamento da cardiomiopatia dilatada. Rev Bras Cir Cardiovasc 2001; 16(4): 275-88.

\section{GRÁFICO 3}

CURVAS DE SOBREVIDA (KAPLAN-MEIER) APÓS A VENTRICULECTOMIA PARCIAL ESQUERDA, DE ACORDO COM CLASSE FUNCIONAL (C.F.). AS PROBABILIDADES SÃO APRESENTADAS COM O LIMITE DE CONFIANCCA DE 70\%. O VALOR DE p SE REFERE AO TESTE DE LOG-RANK

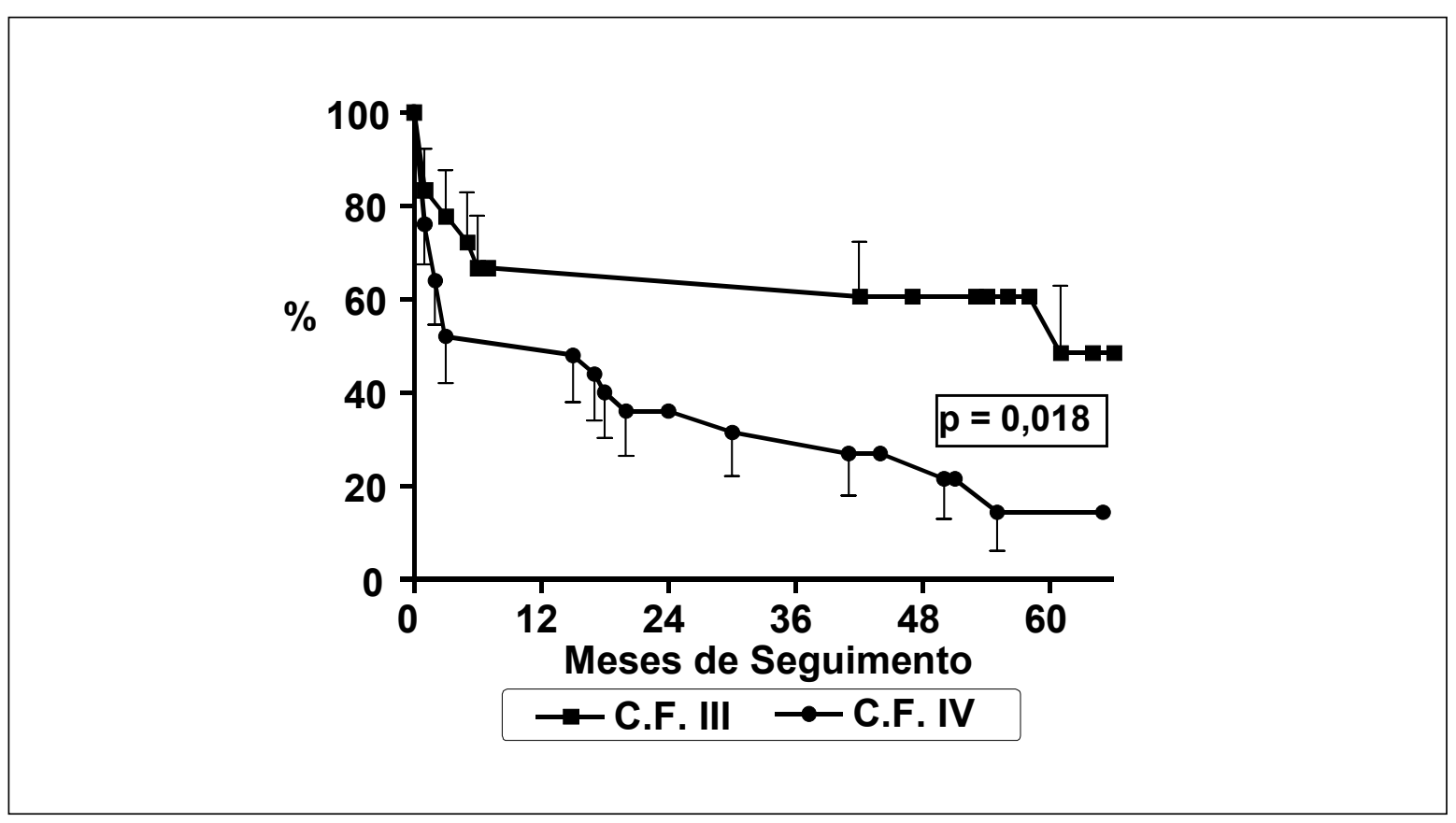

GRÁFICO 4

CURVAS DE SOBREVIDA (KAPLAN-MEIER) APÓS A VENTRICULECTOMIA PARCIAL ESQUERDA, DE ACORDO COM O NÍVEL PLASMÁTICO DE NOR-EPINEFRINA (NOR). AS PROBABILIDADES SÃO APRESENTADAS COM O LIMITE DE CONFIANÇA DE 70\%. O VALOR DE p SE REFERE AO TESTE DE LOG-RANK

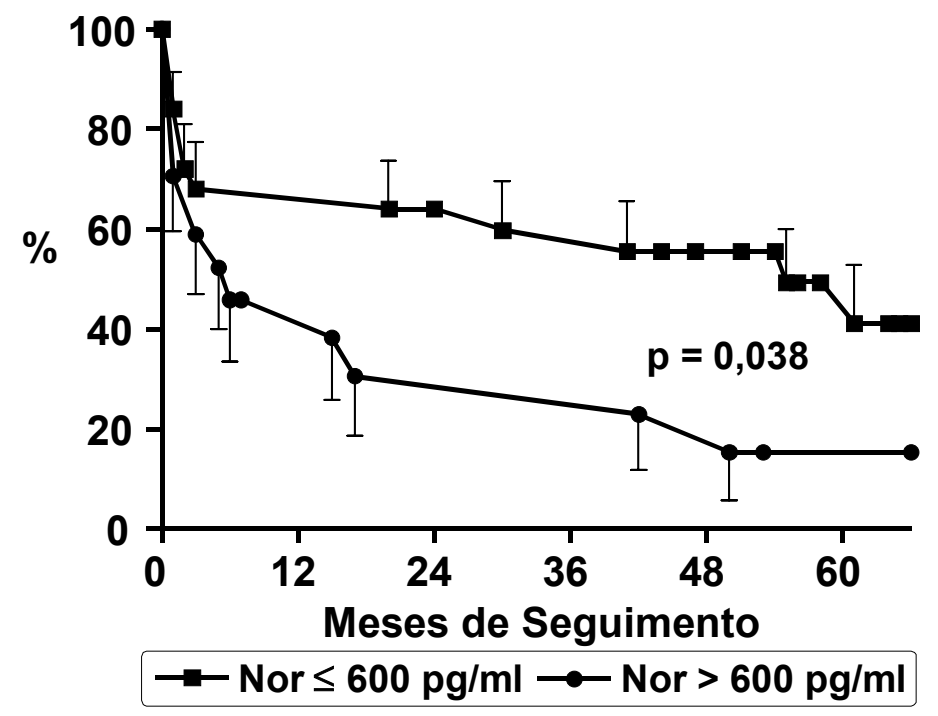


Moreira L F P, Bacal F, Benício A, Bocchi E A, Higuchi M L, Stolf N A G, Oliveira S A - Fatores prognósticos e evolução da função ventricular em 5 anos de seguimento da ventriculectomia parcial esquerda no tratamento da cardiomiopatia dilatada. Rev Bras Cir Cardiovasc 2001; 16(4): 275-88.

\section{GRÁFICO 5}

CURVAS DE SOBREVIDA (KAPLAN-MEIER) APÓS A VENTRICULECTOMIA PARCIAL ESQUERDA, DE ACORDO COM O DIÂMETRO DAS FIBRAS MIOCÁRDICAS (DFM). AS PROBABILIDADES SÃO APRESENTADAS COM O LIMITE DE CONFIANÇA DE 70\%. O VALOR DE p SE REFERE AO TESTE DE LOG-RANK

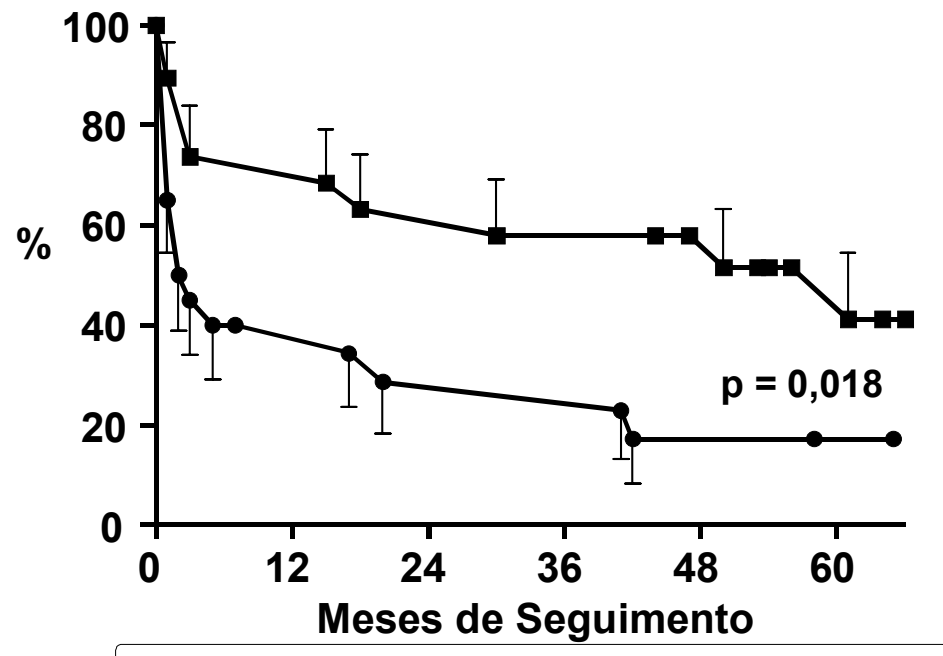

$\rightarrow-D F M \leq 22$ microns $\rightarrow-D F M>22$ microns

\section{GRÁFICO 6}

CURVAS DE SOBREVIDA (KAPLAN-MEIER) APÓS A VENTRICULECTOMIA PARCIAL ESQUERDA, DE ACORDO COM A EXISTÊNCIA DE APOPTOSE DE CÉLULAS MIOCÁRDICAS. AS PROBABILIDADES SÃO APRESENTADAS COM O LIMITE DE CONFIANÇA DE 70\%. O VALOR DE p SE REFERE AO TESTE DE LOG-RANK

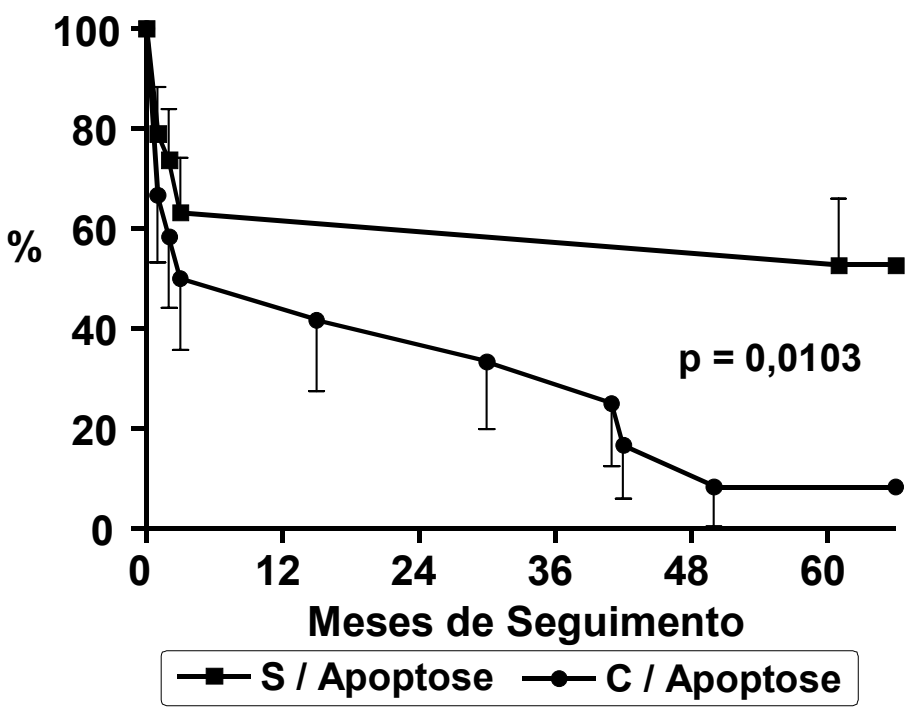


Moreira L F P, Bacal F, Benício A, Bocchi E A, Higuchi M L, Stolf N A G, Oliveira S A - Fatores prognósticos e evolução da função ventricular em 5 anos de seguimento da ventriculectomia parcial esquerda no tratamento da cardiomiopatia dilatada. Rev Bras Cir Cardiovasc 2001; 16(4): 275-88.

TABELA 4

DADOS DA ANGIOGRAFIA COM RADIOISÓTOPOS

\begin{tabular}{lccccccc}
\multicolumn{7}{c}{ DADOS DA ANGIOGRAFIA COM RADIOISÓTOPOS } \\
\hline
\end{tabular}

VDVE, Volume diastólico de ventrículo esquerdo; FEVE, Fração de ejeção de ventrículo esquerdo. Os números entre parêntesis indicam os pacientes avaliados a cada período. * $p<0.05$ em relação valores pré-operatórios obtidos nos mesmos pacientes.

TABELA 5

DADOS DO CATETERISMO CARDÍACO DIREITO

\begin{tabular}{|c|c|c|c|c|c|c|c|}
\hline & \multirow[b]{2}{*}{$\begin{array}{l}\text { Preop. } \\
(n=28)\end{array}$} & \multicolumn{6}{|c|}{ SEGUIMENTO } \\
\hline & & $\begin{array}{c}1 \mathrm{~m} . \\
(\mathrm{n}=28)\end{array}$ & $\begin{array}{l}6 \mathrm{~ms} . \\
(\mathrm{n}=22)\end{array}$ & $\begin{array}{l}12 \mathrm{~ms} \\
(\mathrm{n}=22)\end{array}$ & $\begin{array}{l}24 \mathrm{~ms} . \\
(n=17)\end{array}$ & $\begin{array}{l}36 \mathrm{~ms} . \\
(n=13)\end{array}$ & $\begin{array}{l}48 \mathrm{~ms} \\
(\mathrm{n}=8)\end{array}$ \\
\hline PMAD $(\mathrm{mmHg})$ & $9,7 \pm 4,6$ & $8,2 \pm 4,3$ & $7,5 \pm 4,1$ & $6,8 \pm 4,7^{*}$ & $6 \pm 5,2^{*}$ & $7,7 \pm 6,1$ & $7,8 \pm 4,7$ \\
\hline $\mathrm{PMAP}(\mathrm{mmHg})$ & $36,3 \pm 11$ & $31,8 \pm 10,2^{*}$ & $30,8 \pm 12,2^{*}$ & $32,3 \pm 11,2$ & $33,3 \pm 7,8$ & $31,7 \pm 10,4$ & $29,1 \pm 9,5$ \\
\hline $\mathrm{PMCP}(\mathrm{mmHg})$ & $23,6 \pm 6,8$ & $19,3 \pm 7,1^{*}$ & $19,7 \pm 7,7^{*}$ & $21,3 \pm 7,7$ & $21,7 \pm 6,3$ & $21,7 \pm 7,6$ & $20,3 \pm 7,1$ \\
\hline PAM $(\mathrm{mmHg})$ & $80 \pm 8,5$ & $84,6 \pm 8,6^{*}$ & $92,8 \pm 6,9^{*}$ & $90,6 \pm 7,9^{*}$ & $93,8 \pm 6,3^{*}$ & $95 \pm 7,8^{*}$ & $94,2 \pm 7,6^{*}$ \\
\hline IC $\left(\mathrm{I} / \mathrm{min} \cdot \mathrm{m}^{2}\right)$ & $2,14 \pm 0,47$ & $2,45 \pm 0,51^{*}$ & $2,61 \pm 0,41^{*}$ & $2,59 \pm 0,47^{*}$ & $2,62 \pm 0,44^{*}$ & $2,72 \pm 0,51^{*}$ & $2,47 \pm 0,54$ \\
\hline IS $\left(\mathrm{ml} / \mathrm{m}^{2}\right)$ & $23,9 \pm 6,8$ & $26,6 \pm 8,9^{*}$ & $31,9 \pm 6,4^{*}$ & $32 \pm 7,3^{*}$ & $33,8 \pm 6,8^{*}$ & $35,7 \pm 8,7^{*}$ & $33,7 \pm 7,8$ \\
\hline
\end{tabular}

PMAD, Pressão média de átrio direito; PMAP, pressão média de artéria pulmonar; PMCP, pressão média de capilar pulmonar; PAM, pressão arterial média; IC, índice cardíaco; IS, índice sistólico. Os números entre parêntesis indicam os pacientes avaliados a cada período. * $p<0.05$ em relação valores pré-operatórios obtidos nos mesmos pacientes.

afecções de diversas etiologias nas séries relatadas $(2,7-11,15)$ e pela associação da redução cirúrgica do volume do VE a outros procedimentos, além da correção da insuficiência valvar mitral (2,7-10,15).

Nesta investigação, a ventriculectomia parcial esquerda foi realizada em pacientes portadores de cardiomiopatia dilatada idiopática, sendo associada apenas a eventual correção da insuficiência das valvas atrioventriculares. A evolução clínica dos pacientes foi caracterizada por elevada mortalidade nos primeiros meses de pós-operatório e pela possibilidade de progressão da insuficiência cardíaca e de eventos relacionados a arritmias no seguimento tardio.

Por outro lado, a identificação da influência negativa do comprometimento intrínseco das células miocárdicas sobre os resultados deste procedimento poderia auxiliar na adequada seleção préoperatória dos pacientes, ajudando a estabelecer uma real perspectiva para a ventriculectomia parcial esquerda no tratamento da cardiomiopatia dilatada, como relatado em publicações anteriores ${ }^{(14,16)}$. No entanto, a constatação de que os resultados tardios desta operação também foram influenciados, neste estudo, pela severidade da condição clínica dos pacientes no pré-operatório, impede uma conclusão otimista a respeito de seu emprego clínico.

\section{Modificações da Função Ventricular}

A avaliação de pacientes no primeiro mês de seguimento após a ventriculectomia parcial esquerda tem demonstrado a elevação significativa da fração de ejeção do VE, associada a diminuição do volume ventricular. Esse fato é relatado por vários autores $(4,6,7,13)$, sendo documentado em estudos que utilizaram a ventriculografia com radioisótopos, à semelhança da experiência atual, um aumento médio da fração de ejeção do VE entre $40 \%$ e $70 \%{ }^{(4,6,13)}$. Além dos aspectos já comentados, aumentos significativos do volume sistólico e do débito cardíaco na presença de diminuição das pressões em território capilar pulmonar foram também documentados nos primeiros meses de seguimento após esta operação $(4,6,13,16)$.

A melhora da função ventricular não ocorre, entretanto, em todos os pacientes submetidos a ventriculectomia parcial esquerda. Ausência de elevação da fração de ejeção do VE após a realização do procedimento ocorreu na metade dos pacientes desta investigação e foi associada a elevada mortalidade ou necessidade urgente de transplante cardíaco, nos primeiros meses de seguimento. A esse respeito, o conceito de que a falência miocárdica 
Moreira L F P, Bacal F, Benício A, Bocchi E A, Higuchi M L, Stolf N A G, Oliveira S A - Fatores prognósticos e evolução da função ventricular em 5 anos de seguimento da ventriculectomia parcial esquerda no tratamento da cardiomiopatia dilatada. Rev Bras Cir Cardiovasc 2001; 16(4): 275-88.

é baseada tanto em fatores anatômicos como na existência de lesão das fibras miocárdicas justifica a realização da ventriculectomia parcial, mas também indica que os resultados deste procedimento podem ser limitados pelo grau de comprometimento intrínseco do miocárdio.

O estresse da parede ventricular é um determinante importante do consumo de oxigênio pelo miocárdio e a sua redução, portanto, pode diminuir a demanda miocárdica de oxigênio (17). Em conseqüência, do ponto de vista teórico, além da redução da tensão ventricular e da restituição parcial da contratilidade miocárdica, a ventri-culectomia parcial esquerda pode ser responsável pela interrupção da progressão da cardiomiopatia de base por causa da melhora do metabolismo miocárdico. Esse fato foi sugerido anteriormente pela manutenção dos valores da fração de ejeção do VE e dos parâmetros hemodinâmicos por até dois anos de seguimento em parte dos pacientes deste estudo(14,16). No entanto, a ocorrência de redilatação do VE foi observada após o primeiro ano de pós-operatório, indicando a possibilidade desta técnica se tornar muito mais uma ponte biológica do que uma alternativa real ao transplante cardíaco.

\section{Seguimento Pós-Operatório Imediato}

A mortalidade hospitalar após a ventriculectomia parcial esquerda tem variado entre $3 \%$ e $27 \%$ e tem como causa principal a falência ventricular $(2,4-$ $8,12,16)$, que foi responsável por cerca de $70 \%$ dos óbitos imediatos na experiência aqui relatada. Essa complicação está geralmente associada a falta de melhora da função sistólica do VE após o procedimento e a piora do padrão de restrição diastólica, situação que é diagnosticada pela manutenção de pressões de enchimento elevadas nas câmaras esquerdas. A indicação do balão intra-aórtico, como método de suporte circulatório mecânico, tem sido realizada nesse período em torno de $18 \%$ a $40 \%$ dos pacientes submetidos a ventriculectomia parcial esquerda ${ }^{(4,8,16)}$. Já a utilização de ventrículos artificiais atuando como ponte para a posterior realização de um transplante cardíaco de urgência tem sido descrita em até $16 \%$ dos pacientes no pós-operatório imediato, sendo esse procedimento fundamental para a diminuição da mortalidade hospitalar em centros mais desenvolvidos, como destacado por McCARTHY et al. (12).

Outras causas de óbito no período hospitalar, como o sangramento pela incisão cirúrgica e as arritmias ventriculares sustentadas, também têm sido descritas por vários autores $(3-8,16)$. Embora nem sempre responsáveis pelo óbito dos pacientes, as arritmias ventriculares têm apresentado incidências entre $5 \%$ e $22 \%$ no pós-operatório imediato $(6,16)$.

\section{Seguimento Tardio}

Além da melhora da função ventricular, a ventriculectomia parcial esquerda tem sido também responsável pela melhora da condição clínica dos pacientes sobreviventes na maioria das séries relatadas $(3-6,11,12,15)$. Pacientes submetidos a este procedimento em classe funcional III ou IV geralmente retornam para classe funcional I ou II, fazendo uso de menos medicações do que antes da operação.

Por outro lado, a mortalidade no primeiro ano de seguimento após a ventriculectomia parcial esquerda tem sido elevada na maioria das séries relatadas, exibindo valores entre $32 \%$ e $55 \%(2,3,5-8,15,16)$. Os índices de sobrevida após a ventriculectomia parcial, no entanto, foram semelhantes aos observados em pacientes indicados para o transplante cardíaco na experiência de ETOCH et al. (18), considerando apenas o primeiro ano de seguimento. A mortalidade nos primeiros meses de pós-operatório foi também elevada neste estudo, cujos resultados incluem, adicionalmente, o seguimento dos pacientes até cinco anos de pós-operatório. Os dados encontrados mostram que, no período tardio, apesar da mortalidade ser menor do que nos primeiros meses, os índices de sobrevida após a realização da ventriculectomia parcial foram de apenas $45,9 \%$ em três anos e de $34 \%$ aos cinco anos de seguimento.

A mortalidade no pós-operatório tardio da ventriculectomia parcial esquerda ocorre predominantemente por progressão de insuficiência cardíaca ou por eventos relacionados a arritmias ${ }^{(5-9,12,15,16)}$, fato também observado na atual experiência. Essas complicações apresentam incidências semelhantes após a período hospitalar e têm sido observadas mesmo em experiências que incluem afecções de diversas etiologias e a realização de outros procedimentos associados à ventriculectomia parcial $(5,7-9,11,15)$.

A ventriculectomia parcial esquerda, por outro lado, tem sido seguida em algumas séries da realização de transplante cardíaco de urgência por progressão da insuficiência cardíaca $(4,12)$ ou pelo implante de desfibriladores automáticos (12). Em conseqüência, a avaliação adequada de seus resultados a longo prazo deve também incluir os pacientes submetidos ao transplante cardíaco em caráter de urgência ou que apresentaram episódios de morte súbita ressuscitada como falhas do procedimento. Nesta investigação, esse tipo de análise mostrou que a progressão de insuficiência cardíaca ocorreu em uma incidência de 8,5 eventos por paciente-ano, enquanto que os eventos mórbidos relacionados à arritmias apresentaram uma incidência de 7,3 eventos por 100 paciente-ano, após os seis primeiros meses de pós-operatório. O risco de morte por arritmias nestes pacientes, no entanto, é semelhante ao descrito na literatura para pacientes portado- 
Moreira L F P, Bacal F, Benício A, Bocchi E A, Higuchi M L, Stolf N A G, Oliveira S A - Fatores prognósticos e evolução da função ventricular em 5 anos de seguimento da ventriculectomia parcial esquerda no tratamento da cardiomiopatia dilatada. Rev Bras Cir Cardiovasc 2001; 16(4): 275-88.

res cardiom-iopatias dilatadas em fase avançada (19). A experiência de STARLING et al. ${ }^{(20)}$, por outro lado, mostram índices de falha do procedimento, que foi definida como indicação de transplante cardíaco ou de dispositivos de assistência circulatória mecânica, em torno de $71 \%$ em 30 meses, sendo a progressão da insuficiência cardíaca referida como causa mais importante desse fato.

\section{Fatores Relacionados ao Prognóstico}

A existência de relação significativa entre o diâmetro das fibras miocárdicas e os resultados da ventriculectomia parcial esquerda nos primeiros meses de pós-operatório, em pacientes desta série, foi publicada anteriormente por nós (14). Esse fato confirma que o sucesso deste procedimento depende, principalmente, da existência de um comprometimento anatômico mais importante do que o grau de lesão das células do miocárdio. Resultados semelhantes foram também observados por FRAZIER et al. (21), analisando a experiência conjunta de dois centros de cirurgia cardíaca com o emprego da ventriculectomia parcial em 42 pacientes portadores de cardiomiopatia dilatada. Paralelamente, TERASAKI et al. (22) sugerem a existência de correlação entre a mortalidade após o procedimento e a presença de maior número de células inflamatórias no miocárdio ventricular.

A relação entre o grau de hipertrofia das fibras miocárdicas e a condição clínica de pacientes com insuficiência cardíaca já havia sido observada por UNVERFERTH et al. (23), em 1983. Mais recentemente, esse parâmetro tem sido, inclusive, utilizado para acompanhar a evolução de pacientes submetidos à terapêutica farmacológica (24) ou à assistência circulatória mecânica como ponte para o transplante cardíaco (25).

Em conseqüência, apesar das modificações intrínsecas do miocárdio serem de difícil quantificação pelos métodos convencionais, a avaliação de fragmentos ventriculares obtidos através de técnicas de biópsia endomiocárdica com métodos histológicos e histoquímicos mais refinados pode, potencialmente, contribuir para a seleção adequada dos pacientes para a ventriculectomia parcial. A esse respeito, estudos de autópsia não mostram grande diferença entre o grau de hipertrofia de fibras miocárdicas nos dois lados do septo interventricular (26), justificando a possibilidade da utilização da biópsia endomiocárdica do ventrículo direito. Em relação à análise da função miocárdica, LIM et al. (27) destacam a importância da avaliação da contratilidade de preparações isoladas de miócitos e da medida do cálcio intracelular na determinação das diferenças fisiológicas do miocárdio a nível celular. Paralelamente, além dos métodos de avaliação direta das características intrínsecas do miocárdio ventricular, estudos a respeito da resposta miocárdica ao estresse através da ecocardiografia ou da cintilografia também podem, teoricamente, auxiliar na identificação de pacientes que tenham predomínio dos efeitos anatômicos do remodelamento ventricular e algum grau de reserva contrátil do miocárdio $(28,29)$.

O impacto da identificação de pacientes com comprometimento irreversível do miocárdio sobre os resultados da ventriculectomia parcial esquerda pode ser antecipado pela observação de que pacientes operados com diâmetro médio de fibras miocárdicas abaixo de 22 microns, nesta série, tiveram uma sobrevida de $63,1 \%$ em dois anos e de $51,4 \%$ em cinco anos. Esses valores contrastam positivamente com publicações recentes sobre pacientes avaliados para tratamento cirúrgico da insuficiência cardíaca, que mostram uma probabilidade de sobrevida ao redor de $50 \%$ em dois anos, para pacientes que estavam inicialmente em classe funcional III ou IV (30).

A existência de influência significativa da condição clínica dos pacientes no pré-operatório sobre os resultados tardios da ventriculectomia parcial esquerda neste estudo, por outro lado, mantém aberta a discussão sobre a validade do emprego clínico desta técnica. A observação de índices de sobrevida abaixo de $20 \%$, em cinco anos de seguimento para os pacientes operados em classe funcional IV ou com nível plasmático de nor-epinefrina abaixo de $600 \mathrm{pg} \cdot \mathrm{ml}^{-1}$, sugere a ausência de influência positiva do procedimento analisado sobre a sobrevida desses grupos a longo prazo. Esse fato aponta para a limitação da indicação da ventriculectomia parcial apenas para pacientes que tenham um comprometimento menos severo da função ventricular esquerda. No entanto, esses pacientes têm apresentado, atualmente, uma evolução satisfatória com a otimização da terapêutica medicamentosa ${ }^{(30)}$, ficando a indicação de procedimentos cirúrgicos paliativos restrita apenas àqueles que evoluem com piora progressiva de sua qualidade de vida ou da tolerância ao exercício. A influência da condição clínica pré-operatória dos pacientes sobre os resultados imediatos da ventriculectomia parcial já havia sido sugerida por BESTETTI et al. (3) e por McCARTHY et al. (12), sendo também observada por KAWAGUSHI et al. (9) em dados obtidos em 172 pacientes de um registro multicêntrico internacional.

Apesar de também estar relacionada com um pior prognóstico em pacientes portadores de cardiomiopatia dilatada ${ }^{(31)}$, a identificação da influência da presença de apoptose de células miocárdicas sobre os resultados da ventri- 
Moreira L F P, Bacal F, Benício A, Bocchi E A, Higuchi M L, Stolf N A G, Oliveira S A - Fatores prognósticos e evolução da função ventricular em 5 anos de seguimento da ventriculectomia parcial esquerda no tratamento da cardiomiopatia dilatada. Rev Bras Cir Cardiovasc 2001; 16(4): 275-88.

culectomia parcial, pode ser encarada como uma nova perspectiva para o emprego clínico do procedimento. Esse fato novamente confirma que o sucesso da ventriculectomia parcial parece realmente depender da existência de alterações anatômicas mais importantes do que o grau de comprometimento das fibras miocárdicas.

\section{CONSIDERAÇÕES FINAIS}

O emprego da ventriculectomia parcial esquerda e de outros procedimentos paliativos no tratamento da cardiomiopatia dilatada tem geralmente apresentado resultados limitados. O objetivo principal desses procedimentos, que é a melhora da função ventricular esquerda através da correção parcial de alterações decorrentes do remodelamento ventricular, tem sido alcançado apenas de maneira discreta, não conseguindo bloquear efetivamente os diversos mecanismos fisiopatológicos responsáveis pela progressão da falência cardíaca.

Dessa forma, apesar desses procedimentos serem capazes de melhorar os sintomas de insuficiência cardíaca, os resultados a longo prazo parecem ser limitados pela condição clínica apresentada pelos pacientes no pré-operatório, o que impede a existência de um impacto significativo do tratamento cirúrgico paliativo sobre a sobrevida de pacientes portadores de cardiomiopatias dilatadas. A esse respeito, as terapêuticas farma-cológicas, que também se baseiam na meIhora da condição hemodinâmica dos pacientes, são responsáveis pela reversão transitória dos sintomas de insuficiência cardíaca, mas têm falhado em diminuir significativamente a progressão da cardiomiopatia de base e a mortalidade a longo prazo (17).
Com base nesses fatos, a incorporação definitiva dos procedimentos paliativos no tratamento da cardiomiopatia dilatada depende da melhora de seus resultados e da solução das complicações observadas com essas técnicas a longo prazo. Nesse sentido, a associação de diversos procedimentos, que poderiam atuar com sinergia na melhora ou correção dos vários aspectos anatômico-funcionais envolvidos no processo de remodelamento, é muito atrativa. Paralelamente, o uso de desfibriladores automáticos implantáveis tem sido defendido em associação a qualquer um dos procedimentos paliativos no tratamento da cardiomiopatia dilatada. Em pacientes em fila de espera para o transplante cardíaco, o uso dos desfibriladores é responsável pela diminuição significativa desses eventos, sem contudo influenciar a mortalidade global dos pacientes ${ }^{(32)}$.

A respeito da cirurgia da ventriculectomia parcial esquerda, embora o conceito da influência positiva da redução cirúrgica do volume do VE tenha sido amplamente documentado na literatura, este procedimento, tal como é hoje realizado, apresenta resultados imprevisíveis e de duração limitada. Em conseqüência, o seu emprego clínico permanece restrito àqueles pacientes com contra-indicações efetivas ao transplante cardíaco e que evoluam com sintomas persistentes de insuficiência cardíaca, apesar da otimização da terapêutica medicamentosa.

A possibilidade da identificação de pacientes com maior comprometimento das fibras mio-cárdicas oferece uma perspectiva para a ampliação da utilização desta técnica. No entanto, apenas a potencialização dos resultados observados, associada à prevenção do processo de desadaptação, poderia realmente colocar a redução cirúrgica do volume ventricular como uma alternativa real no tratamento da cardiomiopatia dilatada. 
Moreira L F P, Bacal F, Benício A, Bocchi E A, Higuchi M L, Stolf N A G, Oliveira S A - Fatores prognósticos e evolução da função ventricular em 5 anos de seguimento da ventriculectomia parcial esquerda no tratamento da cardiomiopatia dilatada. Rev Bras Cir Cardiovasc 2001; 16(4): 275-88.

RBCCV 44205-556

Moreira L F P, Bacal F, Benício A, Bocchi E A, Higuchi M L, Stolf N A G, Oliveira S A - Prognostic factors in the follow-up of patients with idiopathic dilated cardiomyopathy submitted to partial left ventriculectomy. Rev Bras Cir Cardiovasc 2001; 16(4): 275-88

ABSTRACT: Objective: Partial left ventriculectomy has been performed in patients with severe cardiomyopathies. The purpose of this investigation is to document the clinical effects of this procedure, associated with mitral insufficiency correction, in 43 patients with idiopathic dilated cardiomyopathy.

Methods: Eighteen patients were in New York Heart Association class III and 25 were in persistent class IV. Seven of these patients were operated on in cardiogenic shock. The procedure was associated with mitral anuloplasty in 32 patients and with mitral replacement in three. Ten patients were also submitted to De Vega tricuspid valve anuloplasty. Automatic cardioverter-defibrillators were implanted in 12 patients.

Results: Nine $(20.9 \%)$ patients died during the hospital period. The follow-up time ranged from two to 57 months, with a mean of 28.3 months. At six months of follow-up, eight patients were in functional class I, 13 patients in class II, three patients in class III e one patient in class IV $(p<0.001)$. On the other hand, nine patients died during the first six months and other ten in the later postoperative period. Actuarial survival rates were $58 ?(? 7 \%$ at one year, $48 ?(? 7 \%$ at two years and 32 (?8\% at five years of follow-up. Stepwise logistic regression demonstrated that six-month survival was significantly affected by the degree of myocytes hypertrophy. The Cox proportional-hazards analysis showed that preoperative functional class and nor-epinephrine levels were significantly associated with a long-term unfavorable outcome. When that analysis also included anatomicopathological variables, the existence of apoptotic myocardial cells and of more important myocytes hypertrophy were identified as the unique independent predictors of poor outcome. Patients operated in functional class III or IV presented survival rates of $60 \pm ? 11 \%$ and of $14(? 8 \%$ at five years, respectively. In the presence of myocardial cells apoptosis, the survival was $8 ?(? 7 \%$ at the same period, where as it was $63(11 \%$ in the absence of that alteration.

Conclusion: Partial left ventriculectomy, associated, when necessary, with mitral insufficiency correction, improves LV function and ameliorates congestive heart failure in patients with idiopathic dilated cardiomyopathy. However, this procedure clinical application is limited by the high mortality observed in the first postoperative months and by the possibility of heart failure progression and of arrhythmia related events at the late follow-up. These facts seem to be influenced by myocardial cells compromise and by patients' preoperative condition.

DESCRIPTORS: Cardiomyopathy, congestive, surgery. Cardiac surgical procedures, methods. Heart ventricle, surgery.

\section{REFERÊNCIAS BIBLIOGRÁFICAS}

1 Batista R J V, Santos J L V, Franzoni M et al. Ventriculectomia parcial: um novo conceito no tratamento cirúrgico das cardiopatias em fase terminal. Rev Bras Cir Cardiovasc 1996; 11: 1-6.

2 Batista R J, Verde J, Nery P et al. - Partial left ventriculectomy to treat end stage heart disease. Ann Thorac Surg 1997; 64: 634-8.

3 Bestetti R B, Moreira-Neto F, Brasil J C, Bombonato R, Sgarbieri R N, Haddad J - Partial left ventriculectomy: preoperative risk factors for perioperative mortality. Int J Cardiol 1998; 67: 143-6.

4 Dowling R D, Koenig S, Laureano M A, Cerrito P, Gray $L A$ - Results of partial left ventriculectomy in patients with end-stage idiopathic dilated cardiomyopathy. J Heart Lung Transplant 1998; 17: 1208-12.

5 Frota Filho J D, Lucchese F A, Blacher C et al. - Três anos de ventriculectomia parcial esquerda: resul- tados globais e tardios em 41 pacientes. Rev Bras Cir Cardiovasc 1999; 14: 75-87.

6 Gradinac S, Miric M, Popovic Z et al. - Partial left ventriculectomy for idiopathic dilated cardiomyopathy: early results and six-month follow-up. Ann Thorac Surg 1998; 66: 1963-8.

7 Isomura T, Suma H, Horii T, Sato T, Kikuchi N, Iwahashi $\mathrm{K}$ - The Batista operation in patients with dilated cardiomyopathy. J Card Surg 1999; 14: 124-8.

8 Izzat M B, Kabbani S S, Suma H, Pandey K, Morishita K, Yim A P C - Early experience with partial left ventriculectomy in the Asia-Pacific region. Ann Thorac Surg 1999; 67: 1703-7.

9 Kawaguchi A T - Partial left ventriculectomy: the first international registry report. Circulation 1999; 100 (Suppl.I): I-168. (Resumo)

10 Kawaguchi A T, Bergsland J, Ishibashi-Ueda $\mathrm{H}$ et al. Partial left ventriculectomy in patients with dilated failing heart. J Card Surg 1998; 13: 335-42. 
Moreira L F P, Bacal F, Benício A, Bocchi E A, Higuchi M L, Stolf N A G, Oliveira S A - Fatores prognósticos e evolução da função ventricular em 5 anos de seguimento da ventriculectomia parcial esquerda no tratamento da cardiomiopatia dilatada. Rev Bras Cir Cardiovasc 2001; 16(4): 275-88.

11 Konertz W, Khoynezhad A, Sidiropoulos A, Borak V, Baumann G - Early and intermediate results of left ventricular reduction surgery. Eur $J$ Cardiothorac Surg 1999; 15(Suppl 1): S26-S30.

12 McCarthy J F, McCarthy P M, Starling R C et al. - Partial left ventriculectomy and mitral valve repair for endstage congestive heart failure. Eur J Cardiothorac Surg 1998; 13: 337-43.

13 Moreira L F P, Stolf N A G, Bocchi E A et al. - Partial left ventriculectomy with mitral valve preservation in the treatment of patients with dilated cardiomyopathy. $J$ Thorac Cardiovasc Surg 1998; 115: 800-7.

14 Stolf N A G, Moreira L F P, Bocchi E A et al. - Determinants of midterm outcome of partial left ventriculectomy in dilated cardiomyopathy. Ann Thorac Surg 1998; 66: 1585-91.

15 Vanelli $\mathrm{P}$, Beretta L, Fundaró $\mathrm{P} M$ et al. - Left ventricular volume reduction for end-stage heart disease. $J$ Card Surg 1999; 14: 60-3.

16 Moreira L F P, Stolf N A G, Bocchi E A et al. - Existe lugar para a ventriculectomia parcial esquerda no tratamento da cardiomiopatia dilatada? Rev Bras Cir Cardiovasc 1998; 13: 89-99.

17 De Keulenaer G W \& Brutsaert D L- Dilated cardiomyopathy: changing pathophysiological concepts and mechanisms of dysfunction. J Card Surg 1999; 14: $64-74$.

18 Etoch S W, Koenig S C, Laureano M A, Cerrito P, Gray $L A$, Dowling R D - Results after left ventriculectomy versus heart transplantation for idiopathic cardiomyopathy. J Thorac Cardiovasc Surg 1999; 117: 952-9.

19 Nagele H \& Rodiger W - Sudden death and tailored medical therapy in elective candidates for heart transplantation. J Heart Lung Transplant 1999; 18: 869-76.

20 Starling R C, McCarthy P M, Hoercher K J, Buda T, Goormastic M, Young J B - Partial left ventriculectomy for dilated cardiomyopathy: a viable option? Circulation 1999; 100 (Suppl.I): I-801. (Resumo)

21 Frazier O H, Gradinac S, Segura A M et al. - Partial left ventriculectomy: which patients can be expected to benefit? Ann Thorac Surg 2000 (No prelo).

22 Terasaki F, Okabe M, Hayashi T et al. - Myocardial inflammatory cell infiltrates in cases of dilated cardiomyopathy: light microscopic, immunohistochemical, and virological analysis of myocardium specimens obtained by partial left ventriculectomy. $J$ Card Surg 1999; 14: 141-6.

23 Unverferth D V, Mehegan J P, Magorien R D, Unverferth B J, Leier C V - Regression of myocardial cellular hypertrophy with vasodilator therapy in chronic congestive heart failure associated with idiopathic dilated cardiomyopathy. Am J Cardiol 1983; 51: 1392-8.

24 Katz A M - Regression of left ventricular hypertrophy: new hope for dying hearts. Circulation 1998; 98: 623-4.

25 Zafeiridis A, Jeevanandam V, Houser S R, Margulies K $B$ - Regression of cellular hypertrophy after left ventricular assist device support. Circulation 1998; 98: 656-62.

26 Unverferth D V, Baker P B, Swift S E et al. - Extent of myocardial fibrosis and cellular hypertrophy in dilated cardiomyopathy. Am J Cardiol 1986; 57: 816-20.

27 Lim K H, Griffiths E J, Pryn S J, Callaway M, Angelini G D - Left ventricular volume reduction: new drawn or false horizon? J Card Surg 1999; 14: 38-45.

28 Merlet $\mathrm{P}$, Benvenuti $\mathrm{C}$, Moyse $\mathrm{D}$ et al. - Prognostic value of MIBG imaging in idiopathic dilated cardiomyopathy. $J \mathrm{NuCl}$ Med 1999; 40: 917-23.

29 Nagaoka H, Isobe N, Kubota S et al. - Myocardial contractile reserve as prognostic determinant in patients with idiopathic dilated cardiomyopathy without overt heart failure. Chest 1997; 111: 344-50.

30 Stevenson L W, Couper G, Natterson B et al. - Target heart failure populations for newer therapies. Circulation 1995; 92 (Suppl II):II 174-81.

31 Saraste A, Pulkki K, Kallajoki M et al. - Cardiomyocyte apoptosis and progression of heart failure to transplantation. Eur J Clin Invest 1999; 29: 380-6.

32 Schmidinger $\mathrm{H}$ - The implantable cardioverter defibrillator as a "bridge to transplant": a viable clinical strategy? Am J Cardiol 1999; 83: 151-7. 